\title{
Estratégias de modelagem da extração de óleos essenciais por hidrodestilação e destilação a vapor
}

\author{
Modeling strategies for essential oil extraction by hydrodistillation and steam distillation
}

\author{
Nathália Viégas Busato $^{\mathrm{I}^{*}}$ Jeniffer Cristina Silveira $^{\mathrm{I}}$ Andréa Oliveira Souza da Costa \\ Esly Ferreira da Costa JuniorI
}

\section{- REVISÃO BIBLIOGRÁFICA -}

\section{RESUMO}

Óleos essenciais são compostos químicos voláteis, característicos por sua fragrância e frequentes atividades antimicrobianas e antioxidantes. São extraídos dos tricomas de plantas aromáticas por meio do processo de destilação, seja ela utilizando apenas vapor (destilação por arraste a vapor), água e vapor ou somente água (hidrodestilação); ou ainda através do processamento mecânico dos pericarpos de frutos cítricos, extração com solventes orgânicos ou com fluido supercrítico, por exemplo. Em ambos os casos, a modelagem matemática é relevante: na destilação a vapor, com o objetivo de projetar plantas industriais com boas condições operacionais; na hidrodestilação, normalmente em escala laboratorial, com o objetivo de se obter uma melhor descrição do comportamento da extração e uma maior precisão na obtenção dos dados experimentais. Neste trabalho, são apresentados, analisados e discutidos quatro modelos do processo de extração.

Palavras-chave: óleos essenciais, difusão intrapartícula, equilíbrio de fases, geometria da partícula.

\section{ABSTRACT}

Essential oils are volatile chemical compounds, characteristic for their fragrance and common antimicrobial and antioxidant activities. These oils are extracted from trichomes of the aromatic plants by distillation process, either using only steam (steam distillation), water and steam or only water (hydrodistillation); or also by mechanical processing of citrus fruit pericarp, extraction with organic solvents or supercritical fluid, for example. In both cases the mathematical modeling is relevant: in the steam distillation in order to design industrial plants with determination optmized operating conditions; in the hydrodistillation, normally used in laboratory scale, in order to obtain a better description of the extraction behavior and a greater accuracy in experimental data determination. In this research four extraction process models from literature are presented, analyzed and discussed.
Key words: essential oils, intraparticle diffusion, phase equilibria, particle geometry.

\section{INTRODUÇÃO}

Os óleos essenciais são compostos principalmente por terpenoides voláteis, que são produzidos pelo metabolismo secundário de plantas aromáticas. Os principais compostos que formam um óleo essencial pertencem às classes químicas dos monoterpenos oxigenados, monoterpenos, sesquiterpenos e sesquiterpenos oxigenados (MEIRELES, 2009). Antigos hieróglifos egípcios e manuscritos chineses relatam que, há mais de mil anos antes de Cristo, os óleos essenciais eram utilizados na cura de algumas doenças (FILIPPIS, 2001).

As principais características de um óleo essencial são sua fragrância e suas atividades antimicrobianas e antioxidantes, portanto, é largamente utilizado em indústrias de perfume, indústrias farmacêuticas, indústrias de cosméticos, dentre outras (SILVEIRA et al., 2012).

Segundo CASSEL et al. (2009), dependendo do método de extração utilizado, a composição química do óleo pode variar significativamente. Podem ser encontrados trabalhos na literatura envolvendo estudos físico-químicos do processo de destilação destes óleos, tais como CERPA et al. (2008), que trata da espécie de planta aromática Lavandula angustifolia L. x latifolia, L., PORNPUNYAPAT et al. (2011), que desenvolvem o

${ }^{\text {I} D e p a r t a m e n t o ~ d e ~ E n g e n h a r i a ~ R u r a l, ~ E n g e n h a r i a ~ Q u i ́ m i c a, ~ U n i v e r s i d a d e ~ F e d e r a l ~ d o ~ E s p i ́ r i t o ~ S a n t o ~(U F E S), ~ C a m p u s ~ d e ~ A l e g r e, ~ A l t o ~}$ Universitário, s/n, CP 16, 29500-000, Guararema, Alegre, ES, Brasil. E-mail: nathalia.busato@hotmail.com.*Autor para correspondência. Recebido 20.12.12 Aprovado 17.02.14 Devolvido pelo autor 02.06.14 CR-2012-1330.R3 
estudo para a espécie Aquilaria crassna, mostrando a variação da composição deste óleo para diferentes temperaturas, BABU \& SINGH (2009), que analisam a composição do óleo de Eucalyptus cinerea e MILOJEVIČ et al. (2008), que estudam a espécie Juniperus communis L.

Conforme a ISO 9235:1997, óleos essenciais são produtos obtidos de partes de plantas através de destilação por arraste a vapor, bem como os produtos obtidos por processamento mecânico dos pericarpos dos frutos cítricos. Segundo GÜNTHER (1948), a região onde se iniciou o processo industrial de destilação de óleos essenciais foi o Oriente, porém, o pleno desenvolvimento das técnicas de destilação ocorreram no Ocidente.

Existem três variações diferentes do processo de destilação com água/vapor: destilação com água (hidrodestilação), destilação com água e vapor e destilação a vapor (ROSTAGNO \& PRADO, 2013). Na hidrodestilação, a planta aromática permanece em contato com a água fervente, podendo estar completamente imersa ou flutuando. Já na destilação com água e vapor, o material a ser destilado é apoiado sobre uma placa perfurada ou inserido em uma rede e colocado logo acima do fundo do destilador. Já na destilação a vapor, a água não é mantida na parte inferior do destilador e o vapor é introduzido através de serpentinas.

Ressalta-se que, no processo de destilação de óleo essencial, é necessário, em alguns casos, realizar fragmentações do material vegetal, com o intuito de facilitar a remoção do óleo dos tricomas glandulares da planta. Este procedimento varia de acordo com a estrutura da planta. Flores, folhas e outras partes finas e não fibrosas não necessitam de fragmentação. As sementes devem ser completamente esmagadas. Raízes, caules e todo o material lenhoso precisam ser cortados em comprimentos curtos (GÜNTHER, 1948). Atenta-se ao fato de que o escoamento em leito fixo envolve alta perda de carga, visto que leitos de partículas finas são compactos e apresentam resistência ao escoamento (KUNII \& LEVENSPIEL, 1991).

Devido à diferença de equipamento e operação na extração por arraste a vapor em laboratório e na indústria, a curva de extração naturalmente não é a mesma. No laboratório, o material vegetal é geralmente pré-tratado antes do processo, ocasionando o aumento da eficiência deste. Nesse caso, uma maneira de se representar melhor uma unidade industrial é a criação de uma unidade piloto, a qual aproxima os dados laboratoriais ao que ocorre efetivamente na indústria de óleos essenciais (SARTOR, 2009).
Desenvolvem-se modelos matemáticos para simular processos industriais, visto que este é um passo inevitável no projeto de plantas industriais com condições operacionais otimizadas (SARTOR, 2009). A maneira pela qual um modelo matemático é desenvolvido permite a sua classificação como fenomenológico (teórico), empírico ou híbrido (PINTO \& LAGE, 2001). Os modelos também podem ser classificados como determinísticos (possuem uma única resposta) ou estocásticos (as respostas dependem de uma distribuição de probabilidade). Quanto à resolução matemática, os modelos podem ser denominados como lineares ou não lineares (THOLON \& QUEIROZ, 2009). Outra classificação útil se refere à possibilidade das propriedades serem uniformes no espaço (parâmetros concentrados) ou não uniformes no espaço (parâmetros distribuídos) (PINTO \& LAGE, 2001).

São encontrados na literatura modelos para o processo de extração de óleos essenciais pertencentes a três categorias: modelos empíricos, modelos baseados no balanço de massa na fase fluida e modelos baseados no balanço de massa na fase sólida (GASPAR et al., 2003).

O objetivo deste trabalho consiste em apresentar, analisar e discutir as estratégias de modelagem da extração de óleos essenciais para os processos de destilação a vapor e hidrodestilação, visto que há semelhanças na modelagem destes dois processos.

Modelos matemáticos

ROMDHANE \& TIZAOUI (2005) propuseram um modelo teórico simplificado para o processo de extração de óleo essencial por arraste a vapor de sementes de erva-doce (Pimpinella anisum). Para tal, os parâmetros de transferência de massa, incluindo o coeficiente de transferência de massa total, foram estimados. As considerações empregadas neste modelo são: o óleo essencial é considerado como formado por um único componente (anetol); a pressão e a temperatura são constantes ao longo da coluna; considera-se que os grãos de ervadoce comportam-se como um material poroso; a transferência de massa é similar à que existe entre um material poroso e um gás; considera-se que não há movimento das partículas devido ao fluxo de vapor; as partículas são esféricas e não alteram sua forma e seu tamanho; inicialmente, a composição do óleo é homogênea; a condensação interna de vapor é desprezível; a difusão de vapor de água no interior dos grãos é negligenciada; considera-se que o sistema é composto por um reator de mistura perfeita. 
A partir das considerações propostas, o balanço de massa total de óleo é dado por:

$$
\mathrm{G}_{\mathrm{g}} \mathrm{y}_{\text {in }}-\mathrm{G}_{\mathrm{g}} \mathrm{y}(\mathrm{t})=\frac{\mathrm{dm}_{\mathrm{ol}}}{\mathrm{dt}}
$$

em que $\mathrm{m}_{\mathrm{ol}}$ é a massa de óleo contido na coluna, $\mathrm{y}_{\text {in }}$ é a fração mássica de óleo essencial no vapor de entrada, y(t) é a fração mássica de óleo essencial no vapor de saída em um tempo t e $\mathrm{G}_{\mathrm{s}}$ é a taxa de fluxo de massa de vapor $\left(\mathrm{kg} \mathrm{s}^{-1}\right)$.

De acordo com ROMDHANE \& TIZAOUI (2005), o vapor que alimenta a coluna é livre de óleo essencial, portanto, $\mathrm{y}_{\mathrm{in}}=0$. Assim:

$$
\mathrm{y}(\mathrm{t})=-\frac{1}{\mathrm{G}_{\mathrm{g}}} \frac{\mathrm{dm}_{\mathrm{ol}}}{\mathrm{dt}}
$$

Considera-se que $\mathrm{M}_{\mathrm{i}}$ é a massa da parte livre de óleo essencial nas sementes e $m_{a s}$ é a massa das sementes. Em qualquer instante t, a relação entre $M_{i}$ e $m_{a s}$ é dada por:

$$
\mathrm{M}_{\mathrm{i}}=\mathrm{m}_{\mathrm{as}}(1-\mathrm{x}(\mathrm{t}))
$$

em que $x(t)$ é a fração de massa de óleo nas sementes no tempo $t$.

A massa de óleo na coluna em um tempo $\mathrm{t}$ é dada pela equacão (4), obtida a partir da substituição da relação de $m_{a s}$ apresentada na equação (3).

$\mathrm{m}_{\mathrm{ol}}(\mathrm{t})=\mathrm{m}_{\mathrm{as}} \mathrm{x}(\mathrm{t})+\varepsilon \rho_{\mathrm{s}} \mathrm{V}_{\mathrm{c}} \mathrm{y}(\mathrm{t})=\mathrm{M}_{\mathrm{i}} \frac{\mathrm{x}(\mathrm{t})}{1-\mathrm{x}(\mathrm{t})}+\varepsilon \rho_{\mathrm{s}} \mathrm{V}_{\mathrm{c}} \mathrm{y}(\mathrm{t})$ em que $\varepsilon$ é a porosidade na coluna, $\rho_{s}$ é a densidade do vapor e $\mathrm{V}_{\mathrm{C}}$ é o volume da coluna.

A diferencial da equação (4) é apresentada na equação (5):

$$
\mathrm{dm}_{\mathrm{ol}}=\mathrm{M}_{\mathrm{i}} \frac{\mathrm{dx}}{(1-\mathrm{x})^{2}}+\varepsilon \rho_{\mathrm{s}} \mathrm{V}_{\mathrm{C}} \mathrm{dy}
$$

Substituindo a equação (5) na equação (2), o balanço de massa é reescrito:

$$
-\mathrm{G}_{\mathrm{s}} \mathrm{ydt}=\mathrm{M}_{\mathrm{i}} \frac{\mathrm{dx}}{(1-\mathrm{x})^{2}}+\varepsilon \rho_{\mathrm{s}} \mathrm{V}_{\mathrm{C}} \mathrm{dy}
$$

ROMDHANE \& TIZAOUI (2005) obtiveram o valor da fração mássica de óleo nas sementes, $\mathrm{x}(\mathrm{t})$, em função da fração mássica de óleo no vapor de saída, $\mathrm{y}(\mathrm{t})$, como mostrado na equação (7), por meio da integração da equação (6) no intervalo de tempo de 0 a t.

$$
x(t)=1-\frac{1-x_{0}}{1-\frac{G_{g}}{m_{\text {aso }}} \int_{0}^{t} y(t) d t+\frac{\Sigma \rho_{g} V_{C}}{m_{\text {aso }}}\left(y_{0}-y\right)}
$$

em que $\mathrm{M}_{\mathrm{i}}=\mathrm{m}_{\text {as0 }}\left(1-\mathrm{x}_{0}\right) ; \mathrm{m}_{\text {as } 0}$ é a massa inicial das sementes, $x_{0}$ é a fração inicial de óleo nas sementes (valor máximo de $\mathrm{x}(\mathrm{t})$ ) e $\mathrm{y}_{0}$ é a fração inicial de vapor de óleo.

A taxa na qual o óleo é transferido para o vapor pode ser descrita da forma:

$$
-\frac{\mathrm{dm}_{\mathrm{ol}}}{\mathrm{dt}}=\mathrm{K} \Omega\left(\mathrm{x}-\mathrm{x}^{*}\right)
$$

em que K é o coeficiente de transferência de massa total, $\Omega$ é a área da superfície disponível para transferência de massa e $x^{*}$ é a fração de óleo nas sementes em equilíbrio com o vapor.
ROMDHANE \& TIZAOUI (2005) assumem que $x^{*}$ é proporcional à pressão de vapor de óleo a uma dada temperatura, portanto:

$$
\mathrm{x}^{*}=\alpha \mathrm{P}^{0}
$$
em que $\alpha$ é o parâmetro de proporcionalidade e $\mathrm{P}^{0}$ é a pressão de vapor do anetol puro. As equações (5) e (8) são combinadas resultando na seguinte equação diferencial:

$\mathrm{M}_{\mathrm{i}} \frac{1}{(1-\mathrm{x})^{2}} \frac{\mathrm{dx}}{\mathrm{dt}}+\varepsilon \rho_{\mathrm{s}} \mathrm{V}_{\mathrm{C}} \frac{\mathrm{dy}}{\mathrm{dt}}=-\mathrm{K} \Omega\left(\mathrm{x}-\alpha \mathrm{P}^{0}\right)$

Considera-se que a área específica de transferência de massa é definida pela expressão a = $\Omega / \mathrm{m}_{\text {as }}$ e a razão entre a coluna de vapor e a massa de material vegetal é definida por $\overline{\mathrm{s}}=\varepsilon \rho_{\mathrm{s}} \mathrm{V}_{\mathrm{c}} / \mathrm{m}_{\mathrm{as}}$. Sendo assim, a equação (10) pode ser reescrita como: $\frac{1-x_{0}}{(1-x)^{2}} \frac{d x}{d t}+\bar{s} \frac{d y}{d t}=-\mathrm{Kax}+\alpha \mathrm{KaP}^{0}$

$$
\text { ROMDHANE \& TIZAOUI }
$$

simplificam a equação (11) considerando que o teor de óleo (x) é pequeno em comparação com o valor 1 $\left(\mathrm{x}_{0}\right.$ é o valor máximo para $\mathrm{x}$ e apresenta valor inferior a 0,03 neste caso). Dessa forma, a equação (11) é reescrita como:

$$
\begin{aligned}
& \frac{\mathrm{dx}}{\mathrm{dt}}+\overline{\mathrm{s}} \frac{\mathrm{dy}}{\mathrm{dt}}=-\mathrm{Kax}+\alpha \mathrm{KaP}^{0} \\
& \text { ROMDHANE \& TIZAOUI }
\end{aligned}
$$

utilizaram uma planta piloto para a coleta dos dados experimentais empregados na estimação dos valores dos parâmetros: Ka e $\alpha$.

Além do estudo de ROMDHANE \& TIZAOUI (2005), existem outros trabalhos que analisam a extração de óleo essencial de sementes com geometria esferoide, assim como BENYOUSSEF et al. (2002), que trata da extração de óleo essencial do coentro (Coriandrum sativum L.). Esses autores verificaram que, tratando-se de sementes com formato esférico, tais modelos tornam-se adequados, porém, de acordo com BENYOUSSEF et al. (2002), esta se torna uma limitação, pois estes modelos são específicos para tal matéria vegetal.

SOVOVÁ \& ALEKSOVSKI (2006) realizaram a modelagem do processo de hidrodestilação, que é constituído por um destilador, um condensador e um separador. O destilador contém partículas da planta imersas em água em ebulição. A fase de vapor, composta por vapor de água e óleo essencial, flui para o condensador, onde há a formação de duas fases, água e óleo, que, logo após, seguem em direção ao separador, onde o óleo, por ser menos denso, flutua na superfície da água. A água retorna da parte inferior do separador de volta para o destilador, podendo conter ainda uma pequena quantidade de óleo essencial. 
Considerou-se um modelo constituído de células rompidas, onde o soluto localizado em suas paredes é rapidamente extraído; e células intactas, onde o soluto se difunde lentamente para a superfície do material vegetal. As considerações utilizadas no modelo são: o óleo essencial é considerado como formado por um pseudo-componente; as partículas de plantas são iguais em tamanho, forma e conteúdo inicial de óleo; nas partículas contidas no destilador, $r$ corresponde à fração de óleo essencial nas células rompidas, facilmente acessíveis, e (1-r) é a fração de óleo essencial nas células intactas; o leito é considerado perfeitamente misturado; a massa de água na fase vapor contida no destilador e no restante dos equipamentos é negligenciada; as resistências à transferência de massa entre as células rompidas e a água fervente e entre a água fervente e a fase vapor são consideradas insignificantes. Desse modo, o equilíbrio é estabelecido instantaneamente entre as fases, de acordo com a equação (13):

$$
\mathrm{x}_{\mathrm{v}}=\mathrm{k}_{\mathrm{v}} \mathrm{x}_{\mathrm{w}}=\mathrm{k}_{\mathrm{v}} \mathrm{k}_{\mathrm{w}} \mathrm{x}_{\mathrm{b}}
$$

em que $x_{v}$ é a concentração mássica de óleo vaporizado, $\mathrm{k}_{\mathrm{v}}$ é o coeficiente de partição vaporlíquido, $\mathrm{x}_{\mathrm{w}}$ é a concentração mássica de óleo na água fervente, $\mathrm{k}_{\mathrm{w}}$ é o coeficiente de partição líquido(células rompidas) e $\mathrm{x}_{\mathrm{b}}$ é a concentração mássica de óleo nas células rompidas.

A concentração inicial no núcleo das partículas compostas por células intactas é igual à concentração de partículas não tratadas, de acordo com a equação (14):

$$
\mathrm{x}_{\mathrm{i}}(\mathrm{t}=0)=\mathrm{x}_{\mathrm{u}}
$$

em que $x_{i}$ é a concentração mássica de óleo nas células intactas e $\mathrm{x}_{u}$ é a concentração mássica de óleo no material não tratado.

A concentração do óleo essencial na corrente que flui a partir do separador de volta para o destilador, $\mathrm{x}_{\mathrm{s}}$, é assumida como sendo constante. $\mathrm{O}$ rendimento $(\mathrm{Y})$ de óleo essencial recolhido no separador é dado por:

$$
Y=\frac{Q}{N} \int_{0}^{t}\left(x_{v}-x_{s}\right) d t
$$

em que $\mathrm{N}$ é a massa da matriz vegetal e $\mathrm{Q}$ é a taxa de vazão de vapor. Ressalta-se que a primeira gota de óleo sai do condensador e segue para o separador em $\mathrm{t}=0$.

Segundo SOVOVÁ \& ALEKSOVSKI (2006), à medida que há o equilíbrio entre as regiões de células rompidas, água fervente e fase vapor, o único processo de transferência de massa que afeta a taxa de destilação de óleo é a difusão de óleo do núcleo da partícula em direção à região de células rompidas.
A transferência de massa a partir das células intactas é simulada como um processo quaseestacionário com fluxo de massa igual a:

$-\{$ volume do núcleo $\} n \rho_{s} \frac{d x_{i}}{d t}=k\{$ superfície do núcleo $\} n \rho_{s}\left(x_{i}-x_{b}\right)$

em que $\mathrm{n}$ é o número de partículas no destilador, $\rho_{s}$ é a densidade real das partículas e $\mathrm{k}$ é o coeficiente de transferência de massa.

Após a introdução da dimensão central característica, definidapor $\lambda=\{$ volume do núcleo $\}$

/\{superfície do núcleo\} , a equação (16) pode ser rearranjada como:

$$
-\frac{\mathrm{dx}_{\mathrm{i}}}{\mathrm{dt}}=\frac{\mathrm{k}}{\lambda}\left(\mathrm{x}_{\mathrm{i}}-\mathrm{x}_{\mathrm{b}}\right)
$$

SOVOVÁ \& ALEKSOVSKI (2006) consideram no modelo dois tipos de partículas: a primeira formada triturando-se um material relativamente isotrópico, como semente ou raiz, e a segunda formada por desintegração de ervas que têm uma parte do óleo essencial depositado em tricomas glandulares, na superfície das suas folhas e outras partes planas e finas.

De acordo com SOVOVÁ \& ALEKSOVSKI (2006), a partícula considerada primeiramente é homogênea em relação à concentração de óleo essencial, mas não no que diz respeito a sua estrutura. Em $\mathrm{t}=0$, uma parte do óleo de fácil acesso, advinda das células rompidas, já foi dissolvida em água fervente. Sendo assim, o balanço de massa para a região com as células rompidas e água fervente é dado por:

$$
\mathrm{rN}\left(\mathrm{x}_{\mathrm{u}}-\mathrm{x}_{\mathrm{b}}\right)=\mathrm{N}_{\mathrm{w}} \mathrm{x}_{\mathrm{w}}
$$

em que $r$ é a fração inicial de óleo de fácil acesso e $\mathrm{N}_{\mathrm{w}}$ é a massa de água no destilador.

A partir da consideração de que as células quebradas, a água fervente e a fase vapor estão em equilíbrio, tem-se que, para $\mathrm{t}=0$ e $\gamma=\mathrm{N}_{\mathrm{w}} / \mathrm{N}$ :

$\mathrm{x}_{\mathrm{i}}=\mathrm{x}_{\mathrm{u}}, \mathrm{x}_{\mathrm{b}}=\frac{\mathrm{r}}{\mathrm{r}+\gamma \mathrm{k}_{\mathrm{w}}} \mathrm{x}_{\mathrm{u}}, \quad \mathrm{x}_{\mathrm{w}}=\frac{\mathrm{k}_{\mathrm{w}} \mathrm{r}}{\mathrm{r}+\gamma \mathrm{k}_{\mathrm{w}}} \mathrm{x}_{\mathrm{u}}$, $\mathrm{x}_{\mathrm{v}}=\frac{\mathrm{k}_{\mathrm{v}} \mathrm{k}_{\mathrm{w}} \mathrm{r}}{\mathrm{r}+\gamma \mathrm{k}_{\mathrm{w}}} \mathrm{x}_{\mathrm{u}}$

Se a partícula for uma esfera de diâmetro $a$ e um diâmetro de núcleo $2 \delta$, o volume do soluto é $(\pi / 6)(2 \delta)^{3}=(1-r)(\pi / 6) a^{3}$. Assim, a relação entre as dimensões do núcleo e da partícula é $2 \delta=(1-r)^{1 / 3} a$, a superfície do núcleo é $\pi(1-r)^{2 / 3} a^{2}$ e a dimensão característica do núcleo é:

$$
\begin{aligned}
& \lambda=\frac{\text { \{volume do núcleo\} }}{\text { \{superficie do núcleo\} }} \frac{a}{6}(1-r)^{\frac{1}{s}} \\
& \text { SOVOVÁ \& ALEKSOVSKI (2006) }
\end{aligned}
$$

consideraram, para o segundo tipo de partícula, uma folha em que os tricomas glandulares são 
representados por uma linha grossa sobre a superfície superior desta. Inicialmente, tem-se que $r$ é a fração do óleo essencial em tricomas glandulares e (1-r) é a fração de óleo uniformemente distribuída no interior da folha (células intactas). Presume-se que os tricomas glandulares rompem logo que a água entra em ebulição e o óleo contido neles é transferido para a água. Portanto, as condições iniciais são:

$\mathrm{x}_{\mathrm{i}}=(1-\mathrm{r}) \mathrm{x}_{\mathrm{u}}, \mathrm{x}_{\mathrm{w}}=\frac{\mathrm{r}}{\gamma} \mathrm{x}_{\mathrm{u}}, \mathrm{x}_{\mathrm{v}}=\frac{\mathrm{rk}_{\mathrm{v}}}{\gamma} \mathrm{x}_{\mathrm{u}}$

Como o volume do núcleo é $2 \delta a^{2}$ e a superfície é $2 a(a+4 \delta)$, a dimensão característica do núcleo é a seguinte:

$$
\lambda=\frac{\delta}{1+4 \delta / a}
$$

Segundo SOVOVÁ \& ALEKSOVSKI (2006), para partículas suficientemente grandes $(a>>\delta)$, a dimensão característica do núcleo tornase independente do tamanho da partícula, $\lambda=\delta$ À medida que o núcleo ocupa todo o volume da partícula e o soluto é transferido do núcleo para a água, a concentração da fase sólida na superfície das partículas é igual a $\mathrm{x}_{\mathrm{w}} / \mathrm{k}_{\mathrm{w}}$ e a equação (17) é reescrita da seguinte forma:

$$
-\frac{\mathrm{dx}_{\mathrm{i}}}{\mathrm{dt}}=\frac{\mathrm{k}}{\lambda}\left(\mathrm{x}_{\mathrm{i}}-\frac{\mathrm{x}_{\mathrm{w}}}{\mathrm{k}_{\mathrm{w}}}\right)
$$

$\mathrm{O}$ balanço de massa para o óleo essencial no destilador, extraído de partículas como as do segundo tipo, é dado por:

$$
(1-\mathrm{r}) \mathrm{N} \frac{\mathrm{dx_{i }}}{\mathrm{dt}}+\mathrm{rN} \frac{\mathrm{dx}_{\mathrm{b}}}{\mathrm{dt}}+\mathrm{N}_{\mathrm{w}} \frac{\mathrm{dx}_{\mathrm{w}}}{\mathrm{dt}}+\mathrm{Q}\left(\mathrm{x}_{\mathrm{v}}-\mathrm{x}_{\mathrm{s}}\right)=0
$$

SOVOVÁ \& ALEKSOVSKI (2006) ressaltaram em seu trabalho que o modelo descrito é somente aplicado a plantas aromáticas imersas em água (hidrodestilação). Para a estimação dos parâmetros do modelo, os autores utilizaram dados experimentais de folhas rasteiras de tomilho (Thymus vulgaris) e sementes intactas de coentro (Coriandrum sativum).

XAVIER et al. (2011) realizaram a modelagem matemática do processo de destilação a vapor do óleo essencial de três espécies de Baccharis, baseada nas discussões apresentadas em SOVOVÁ (2005), cujas considerações se assemelham às de SOVOVÁ \& ALEKSOVSKI (2006). Em sua conclusão, XAVIER et al. (2011) discutem a versatilidade de seu modelo, visto que inclui parâmetros como o coeficiente de transferência de massa e a constante de equilíbrio, além de considerarem a difusão interna nas partículas, evidenciando assim os diferentes efeitos percebidos no processo. Dessa forma, XAVIER et al. (2011) mostram que as hipóteses apresentadas em seu modelo descrevem melhor a realidade observada, em comparação com modelos que se restringem apenas à difusão, como é o caso dos trabalhos de CASSEL \& VARGAS (2006) e CASSEL et al. (2009), que serão descritos a seguir.

CASSEL \& VARGAS (2006) modelaram a extração de óleo essencial por arraste a vapor para a citronela (Cymbopogon winterianus) e CASSEL et al. (2009) utilizaram o alecrim (Rosmarinus officinalis L.), o manjericão (Ocimum basilium L.) e a lavanda (Lavandula dentata L.) em seus estudos, baseados na difusão na fase sólida (folha da planta). Nesse caso, trata-se de um modelo a parâmetros distribuídos e o perfil de concentração dentro da fase sólida é considerado, tendo o coeficiente de difusão como parâmetro a ser ajustado.

A partir do processo de extração realizado em escala de bancada, CASSEL \& VARGAS (2006) e CASSEL et al. (2009) coletaram dados experimentais de rendimento de extração de óleos essenciais para posterior comparação com o modelo matemático.

No desenvolvimento do modelo, CASSEL \& VARGAS (2006) e CASSEL et al. (2009) assumem as seguintes considerações: a etapa controladora do processo de transferência de massa do óleo das folhas para o vapor é a difusão no interior das folhas; a distribuição do óleo essencial em todas as folhas é homogênea no início do processo de extração; considera-se, a variação da concentração ao longo da espessura (L) da folha (unidimensional), existe uma simetria em relação à espessura da mesma (variável x), sendo assim, os cálculos são referentes somente a um dos contornos; a lei de Fick da difusão modela o processo difusivo; após o início da extração, não há resistência à transferência de massa da superfície da planta para o vapor e a concentração de óleo na fase vapor é muito baixa e considerada nula.

A partir das considerações acima, o balanço de massa na fase sólida, equação (25), e suas condições iniciais e de contorno são dados por:

$$
\begin{aligned}
& \frac{\partial^{2} \mathrm{C}_{\mathrm{a}}(\mathrm{x}, \mathrm{t})}{\partial \mathrm{x}^{2}}=\frac{1}{\mathrm{D}} \frac{\partial \mathrm{C}_{\mathrm{a}}(\mathrm{x}, \mathrm{t})}{\partial \mathrm{t}} \\
& \mathrm{C}_{\mathrm{a}}(\mathrm{x}, 0)=\mathrm{C}_{\mathrm{a} 0} ; \mathrm{C}_{\mathrm{a}}(0, \mathrm{t})=0 ; \mathrm{C}_{\mathrm{a}}(\mathrm{L}, \mathrm{t})=0
\end{aligned}
$$

em que $C_{a}(x, t)$ é a concentração de óleo (agrupado com um único pseudocomponente) na fase sólida, D é o coeficiente de difusão do óleo na fase sólida, $\mathrm{C}_{\mathrm{a}}$ é a concentração inicial de óleo essencial na planta aromática e L é a espessura da planta.

Visto que os modelos de CASSEL \& VARGAS (2006) e CASSEL et al. (2009) assumem que o coeficiente de difusão é constante, o modelo apresentado possui solução analítica dada pela equação (27).

$C_{a}(x, t)=\sum_{n=1}^{\infty} \frac{4 C_{a 0}}{n \pi} \operatorname{sen}\left(\frac{n \pi}{L} x\right) e^{-D\left(\frac{n \pi}{L}\right)^{2} t}$ 
SILVEIRA et al. (2012) verificaram, a partir da implementação da equação (27) no software MATHCAD 14.0, que ocorreu um equívoco na apresentação da solução analítica do modelo de CASSEL \& VARGAS (2006) e CASSEL et al. (2009). Os resultados obtidos com tal implementação são valores de concentração de óleo essencial na folha no início do processo, entretanto, não correspondem à concentração inicial de óleo na folha. Além disso, aponta-se a inexistência de simetria em relação à espessura da folha, sendo essas observações, portanto, incompatíveis com as características do problema.

A equação corrigida, apresentada em SILVEIRA et al. (2012), é dada a seguir:

$C_{a}(x, t)=\sum_{m=0}^{\infty} \frac{4 C_{a 0}}{(2 m+1) \pi} \operatorname{sen}\left(\frac{(2 m+1) \pi}{L} x\right) e^{\left(\frac{(2 m+1)^{2} \pi^{2} D}{L^{2}} t\right)}$

A lei de Fick da difusão enuncia que o fluxo difusivo $\left(\overrightarrow{J_{a}}\right)$ é proporcional à derivada da concentração com a direção espacial, conforme equação (29).

$$
\overrightarrow{\mathrm{J}_{\mathrm{a}}}=-\mathrm{D} \frac{\mathrm{dC}_{\mathrm{a}}}{\mathrm{dx}}
$$

Para calcular a taxa de massa extraída, CASSEL \& VARGAS (2006) e CASSEL et al. (2009) utilizam a seguinte equação:

$$
\dot{\mathrm{m}}(\mathrm{t})=\frac{4 \mathrm{C}_{\mathrm{ag}} \mathrm{DA}}{\mathrm{L}} \sum_{\mathrm{m}=0}^{\infty} \mathrm{e}^{-\mathrm{D}\left(\frac{(2 \mathrm{~m}+1) \pi}{\mathrm{L}}\right)^{2}} \mathrm{t}
$$

em que $\dot{m}(\mathrm{t})$ é a taxa de massa extraída como função do tempo e A é a área superficial.

A massa extraída como função do tempo é dada pela integração da equação (30) e é apresentada na equação (31).

$$
\mathrm{m}(\mathrm{t})=\frac{8 \mathrm{~m}_{\mathrm{g} 0}}{\pi^{2}} \sum_{\mathrm{m}=0}^{\infty} \frac{1-e^{-\frac{(2 \mathrm{~m}+1)^{2} \pi^{2} \mathrm{Dt}}{\mathrm{L}^{2}}}}{(2 \mathrm{~m}+1)^{2}}
$$

em que $m(t)$ é a massa extraída como função do tempo e $\mathrm{m}_{\mathrm{a} 0}$ é a massa inicial de óleo contida em todas as plantas. Ressalta-se que $\mathrm{m}_{\mathrm{a} 0}$ é o produto do volume (área de um dos contornos $(=\mathrm{A} / 2)$ vezes a espessura, L) pela concentração inicial de óleo essencial nas plantas $\left(\mathrm{C}_{\mathrm{a} 0}\right)$.

O grau de extração de óleo é então definido pela equação (32):

$$
\mathrm{e}(\mathrm{t})=\frac{\mathrm{m}(\mathrm{t})}{\mathrm{m}(\infty)}=\frac{\sum_{\mathrm{m}=0}^{\infty} \frac{\mathrm{e}^{-\frac{(2 \mathrm{~m}+1)^{2} \pi^{2} \mathrm{Dt}}{\mathrm{L}^{2}}}}{\sum_{\mathrm{m}=0}^{\infty} \frac{1}{(2 \mathrm{~m}+1)^{2}}}}{{ }^{2}}
$$

CASSEL \& VARGAS (2006) e CASSEL et al. (2009) utilizaram dados experimentais de grau de extração em função de tempo e a equação (32) para a estimação do coeficiente de difusão do óleo essencial na fase sólida.
SARTOR (2009) e SARTOR et al. (2011) desenvolveram um modelo para a destilação a vapor, baseado no estudo proposto por REVERCHON (1996) para a extração por fluido supercrítico, visto que, segundo REVERCHON \& MARRONE (1997), pode-se extrapolar este modelo para o caso da extração por arraste a vapor, ressaltando-se que a validade destes varia de acordo com a estrutura do material vegetal utilizado.

As considerações propostas por SARTOR (2009) e SARTOR et al. (2011) são: o óleo extraído considerado como sendo representado por um pseudocomponente; o vapor d'água é uniformemente distribuído nas seções radiais do extrator e sua velocidade é considerada constante; a porosidade do leito não é afetada pela compactação deste; o equilíbrio de fases é dado por uma correlação linear da concentração de soluto; as concentrações de óleo no fluido e no sólido dependem do tempo de extração e da coordenada axial (z), sendo que esta representa a dimensão espacial ao longo do reator; a dispersão axial foi negligenciada; a temperatura e a pressão são consideradas constantes durante a extração.

A partir das considerações acima, tem-se o equacionamento composto pelos balanços de massa na fase vapor e na fase sólida, equações (33) e (34), e suas condições iniciais e de contorno, dados por:

$$
\begin{aligned}
& \frac{\partial \mathrm{C}(\mathrm{z}, \mathrm{t})}{\partial \mathrm{t}}=-v \frac{\partial \mathrm{C}(\mathrm{z}, \mathrm{t})}{\partial z}-\frac{(1-s)}{s} \rho_{s} \frac{\partial \mathrm{q}(\mathrm{z}, \mathrm{t})}{\partial \mathrm{t}} \\
& \frac{\partial \mathrm{q}(\mathrm{z}, \mathrm{t})}{\partial \mathrm{t}}=-\kappa_{\mathrm{TM}}\left[\mathrm{q}(\mathrm{z}, \mathrm{t})-\mathrm{q}^{*}(\mathrm{z}, \mathrm{t})\right] \\
& \mathrm{C}(\mathrm{z}, 0)=0 ; \mathrm{q}(\mathrm{z}, 0)=\mathrm{q}_{0} ; \mathrm{C}(0, \mathrm{t})=0
\end{aligned}
$$

em que $C(z, t)$ é a concentração de óleo na fase fluida, $q(z, t)$ é a concentração de óleo na planta aromática (que varia com a posição axial do reator, mas não ao longo da espessura da planta, visto que se assume um modelo a parâmetros concentrados na fase sólida); $\varepsilon$ é a porosidade do leito; $\mathrm{k}_{\mathrm{TM}}$ é o coeficiente de transferência de massa na fase sólida; $\mathrm{q}_{0}$ é a concentração inicial de óleo nas plantas; e $\mathrm{q}^{*}(\mathrm{z}, \mathrm{t})$ é a concentração de óleo na planta aromática em equilíbrio com a concentração de óleo na fase vapor, assumida por SARTOR (2009) e SARTOR et al. (2011) como sendo linear conforme a equação (36).

$$
\mathrm{q}^{*}(\mathrm{z}, \mathrm{t})=\mathrm{K}_{\mathrm{eq}} \mathrm{C}(\mathrm{z}, \mathrm{t})
$$

Ressalta-se que, nos trabalhos de SARTOR (2009) e SARTOR et al. (2011), há um equívoco na apresentação da equação (33), já que a porosidade não aparece no denominador do primeiro termo à direita da igualdade, conforme apresentado em BUSATO et al. (2012). No entanto, no programa computacional apresentado em SARTOR (2009), este equívoco é corrigido e, portanto, não há erros nos resultados numéricos apresentados pelos autores. 
De acordo com SARTOR (2009) e SARTOR et al. (2011), para a determinação da quantidade total de óleo extraído, é necessário integrar a massa do óleo que sai do extrator em todo o tempo de operação, como representado na equação a seguir:

$$
\frac{\partial \mathrm{m}(\mathrm{t})}{\partial \mathrm{t}}=\mathrm{Q} \cdot \mathrm{C}(\mathrm{H}, \mathrm{t})
$$

em que m(t) é a massa de óleo extraída do reator, Q é a vazão volumétrica de vapor e $\mathrm{H}$ é a altura do leito de extração. A condição inicial para m(t) é:

$$
\mathrm{m}(0)=0
$$

Para a solução do modelo descrito, SARTOR (2009) e SARTOR et al. (2011) realizaram a discretização da direção espacial z para as equações (33) e (34), por diferenças finitas com $\mathrm{N}$ pontos de discretização, gerando $2 \mathrm{~N}$ equações diferenciais ordinárias, que, somadas à equação (37), formam um sistema de $2 \mathrm{~N}+1$ EDOs, que foram integrados no tempo de operação do extrator a partir do software DASSLC (Differential-Algebraic System Solver in C). A simulação dinâmica e a estratégia de estimação de parâmetros foram implementados no software EMSO (Environment for Modeling, Simulation and Optimization).

SARTOR (2009) aplicou seu modelo à extração de óleo essencial de alecrim (Rosmarinus officinalis L.) e de citronela (Cymbopogon winterianus) e SARTOR et al. (2011) aplicou-o à extração de alecrim apenas, sendo que os dados industriais utilizados foram coletados para diferentes estações do ano (verão e inverno). A partir destes dados, foram estimados os seguintes parâmetros: coeficiente global de transferência de massa $\left(\mathrm{K}_{\mathrm{TM}}\right)$ e a constante de equilíbrio $(\mathrm{K})$, para ambas as plantas.

Além dos modelos descritos neste trabalho, podem-se citar também outros estudos que envolvem a hidrodestilação, tais como PORNPUNYAPAT et al. (2011), BABU \& SINGH (2009), MILOJEVIČ et al. (2008) e a destilação a vapor, como os de XAVIER et al. (2011), CERPA et al. (2008), TAJJUDIN et al. (2009). Existem trabalhos que envolvem os dois processos em seus estudos, dentre eles, pode-se citar BOUTEKEDJIRET et al. (2003).

\section{CONCLUSÃO}

Levando-se em consideração os estudos da literatura sobre a extração de óleos essenciais, nota-se que existem, pelo menos, três fenômenos que controlam a hidrodestilação e a destilação a vapor, sendo eles: a vaporização instantânea do óleo na interface da película formada na superfície do material vegetal e o vapor circundante, a transferência de óleo na corrente de vapor circundante e a exsudação do óleo desde o interior dos tricomas glandulares, através de sua cutícula, até a película superficial do material vegetal.

No processo de modelagem, via de regra, tem-se a adoção de considerações para a simplificação dos modelos. Dos modelos apresentados e discutidos neste trabalho, há aqueles que consideram apenas o transporte de massa como sendo a etapa controladora do processo. No entanto, há aqueles que, além de considerarem a difusão intrapartícula, empregam também o equilíbrio entre fases em seus equacionamentos.

Deuma maneira geral, estratégias diferentes são aplicadas na modelagem de processos de extração de óleos essenciais, de acordo com as características que se quer enfatizar, visto que, cada modelo possui particularidades que determinam sua complexidade. Um exemplo peculiar é o trabalho de BENYOUSSEF et al. (2002), que realiza a modelagem utilizando considerações aplicadas apenas a frutos de geometria esférica, ou seja, para tais frutos, o modelo se adequa bem aos dados experimentais, porém, isso se torna uma limitação caso haja a necessidade de modelar o processo envolvendo um material vegetal com diferente geometria. Em contrapartida, SOVOVÁ \& ALEKSOVSKI (2006) apresentam um modelo adaptável tanto para sementes esféricas, quanto para folhas. Portanto, o modelo adequado ao problema é aquele que leva em consideração suas especificidades, ressaltando-se que modelos generalizados, apesar de apresentarem menor precisão, possuem maior aplicabilidade.

\section{AGRADECIMENTOS}

Ao Conselho Nacional de Desenvolvimento Científico e Tecnológico $(\mathrm{CNPq})$, pela bolsa DTI, e à Fundação de Amparo à Pesquisa do Espírito Santo (FAPES), pelas bolsas de iniciação científica e pela bolsa Pesquisador Capixaba.

\section{REFERÊNCIAS}

BABU, G.D.K.; SINGH, B. Simulation of Eucalyptus cinerea oil distillation: A study on optimization of 1,8-cineole production. Biochemical Engineering Journal, v.44, p.226-231, 2009. Disponível em: <http://dx.doi.org/10.1016/j.bej.2008.12.012>. Acesso em: 13 dez. 2012. doi:10.1016/j.bej.2008.12.012.

BENYOUSSEF, E.H. et al. Modélisation du transfert de matiére lors de l'extraction de l'huile essentielle des fruits de coriandre. Chemical Engineering Journal, v.85, p.1-2, 2002. Disponível em: <http://dx.doi.org/10.1016/S1385-8947(01)00134-6>. Acesso em: 13 mar. 2013. doi 10.1016/S1385-8947(01)00134-6. 
BOUTEKEDJIRET, C. et al. Extraction of rosemary essential oil by steam distillation and hydrodistillation. Flavour and Fragrance Journal, v.18, n.6, p.481-484, 2003. Disponível em: $<\mathrm{http} / / / \mathrm{dx}$.doi.org/10.1002/ffj.1226>. Acesso em: 11 fev. 2013. doi: $10.1002 /$ ffj. 1226 .

BUSATO, N.V. et al. Proposta de modelagem da extração de óleos essenciais utilizando modelo a parâmetros agrupados na fase sólida. In: ENCONTRO LATINO AMERICANO DE INICIAÇÃO CIENTÍFICA, 16., 2012, São José dos Campos, SP. Anais... São José dos Campos: Universidade do Vale do Paraíba, 2012. CD-ROM.

CASSEL, E. et al. Steam distillation modeling for essential oil extraction process. Industrial Crops and Products, v.29, p.171-176, 2009. Disponível em: <http://dx.doi.org/10.1016/j. indcrop.2008.04.017>. Acesso em: 9 set. 2012. doi: 10.1016/j. indcrop.2008.04.017.

CASSEL, E.; VARGAS, R.M.F. Experiments and modeling of the Cymbopogon winterianus essential oil extraction by steam distillation. Revista de la Sociedad Química de México, v.50, p.126-129, 2006 .

CERPA, M.G. et al. Modelling steam distillation of essential oils: aplication to lavandin super oil. AIChE Journal, v.54, n.4, p.909917, 2008. Disponível em: <http://dx.doi.org/10.1002/aic.11438>. Acesso em: 15 abr. 2013. doi 10.1002/aic.11438.

FILIPPIS, F.M. Extração com $\mathrm{CO}_{2}$ supercrítico de óleos essencial de Hon-sho e Ho-sho- experimentos e modelagem. 2001. 114f. Dissertação (Mestrado em Engenharia Química) Departamento de Engenharia Química, Universidade Federal do Rio Grande do Sul, RS.

GASPAR, F. et al. Modelling the extraction of essential oils with compressed carbon dioxide. Journal of Supercritical Fluids, v.25, p.247-260, 2003. Disponível em: <http://dx.doi.org/10.1016/ S0896-8446(02)00149-3>. Acesso em: 13 dez. 2012. doi: 10.1016/S0896-8446(02)00149-3.

GÜNTHER, E. History and origin in plants production analysis. The essential oils. New York: Krieger Publishing, 1948. p 235-240.

INTERNATIONALORGANIZATION FOR STANDARDIZATION Aromatic natural raw materials - Vocabulary. ISO 9235:1997. Genebra, 1997. 14p.

KUNII, D.; LEVENSPIEL, O. Fluidization engineering. 2.ed. USA: Butterworth-Heinemann, 1991. 491p.

MEIRELES, M.A.A. Extracting bioactive compounds for food products: theory and applications. Boca Ranton: CRC, 2009. 464p.

MILOJEVIČ, S.Z. et al. Kinetic of distillation of essential oil from comminuted ripe juniper (Juniperus communis L.) berries. Biochemical Engineering Journal, v.39, p.547-553, 2008 Disponível em: <http://dx.doi.org/10.1016/j.bej.2007.10.017>. Acesso em: 17 jun. 2013. doi:10.1016/j.bej.2007.10.017.

PINTO, J.C.; LAGE, P.L.C. Métodos numéricos em problemas de engenharia química. Rio de Janeiro: e-papers, 2001. 281p.
PORNPUNYAPAT, J. et al. Mathematical modeling for extraction of essential oil from Aquilaria crassna by hydrodistillation and quality of agarwood oil. Bangladesh J. Pharmacol, v. 6, p. 18-24, 2011. doi: 10.3329/bjp.v6i1.7902.

REVERCHON, E.; MARRONE, C. Supercritical extraction of clove bud essential oil: isolation and mathematical modeling. Chemical Engineering Science, v.52, n.20, p.3421-3428, 1997. Disponível em: <http://dx.doi.org/10.1016/S00092509(97)00172-3>. Acesso em: 10 nov. 2012. doi: 10.1016/ S0009-2509(97)00172-3.

REVERCHON, E. Mathematical modeling of supercritical extraction of sage oil. American Institute of Chemical Engineers Journal, v.42, n.6, p.1765-1771, 1996.

ROMDHANE, M.; TIZAOUI, C. The kinetic modeling of a steam distillation unit for the extraction of aniseed (Pimpinella anisum) essential oil. Journal of Chemical Technology and Biotechnology, v.80, p.759-766, 2005. doi: 10.1002/jctb.1221.

ROSTAGNO, M.A.; PRADO, J.M. Natural product extraction: principles and applications. Londres: Royal Society of Chemistry, 2013. 500p

SARTOR, R.B. et al. Dynamic simulation of rosemary essential oil extraction in an industrial steam distillation unit. Industrial \& Engineering Chemistry Research, v.50, p.3955-3959, 2011. doi: 10.1021/ie1015848.

SARTOR, R.B. Modelagem, simulação e otimização de uma unidade industrial de extração de óleos essenciais por arraste a vapor. 2009. 99f. Dissertação (Mestrado em Pequisa e Desenvolvimento de Processos) - Escola de Engenharia, Universidade Federal do Rio Grande do Sul, RS.

SILVEIRA, J.C. et al. Levantamento e análise de métodos de extração de óleos essenciais. Enciclopédia Biosfera, v.8, n.15, p.2038-2052, 2012.

SILVEIRA, J.C. et al. Proposta de modelagem da extração de óleos essenciais utilizando modelo a parâmetros distribuídos na fase sólida. In: ENCONTRO LATINO AMERICANO DE INICIAÇÃO CIENTÍFICA, 16., 2012, São José dos Campos, SP. Anais... São José dos Campos: Universidade do Vale do Paraíba, 2012. CD-ROM

SOVOVÁ, H.; ALEKSOVSKI, S.A. Mathematical model for hydrodistillation of essential oils. Flavour and Fragrance Journal, v. 21, p.881-889, 2006. Disponível em: <http://dx.doi. org/10.1002/ffj.1729> . Acesso em: 26 out. 2012. doi: 10.1002/ ffj.1729.

SOVOVÁ, H. Mathematical model for supercritical fluid extraction of natural products and extraction curve evaluation. journal Supercrit Fluids, v.33, p.35-52, 2005. Disponível em: $<\mathrm{http} / / / \mathrm{dx}$.doi.org/10.1016/j.supflu.2004.03.005>. Acesso em: 26 out. 2012. doi: 10.1016/j.supflu.2004.03.005.

TAJJUDIN, N. et al. Model predictive control for steam distillation essential oil extraction process. IEEE STUDENT CONFERENCE ON RESEARCH AND DEVELOPMENT, 2009, Malaysia. Proceedings... UPM Serdang 2009. Disponível em: $<$ http://dx.doi. org/10.1109/SCORED.2009.5442977>. Acesso em: 14 mar. 2013. doi: 10.1109/SCORED.2009.5442977. 
THOLON, P.; QUEIROZ, S.A. Modelos matemáticos utilizados para descrever curvas de crescimento em aves aplicados ao melhoramento genético animal. Ciência Rural, v.39, n.7, p.22612269, 2009. Disponível em: <http://dx.doi.org/10.1590/S0103$84782009000700050>$. Acesso em: 4 jun. 2013. doi: 10.1590/ S0103-84782009000700050.
XAVIER, V.B. et al. modeling for extraction of essential oil from Baccharis spp. by steam distillation. Industrial Crops and Products, v.33 p.599-604, 2011. Disponível em: <http://dx.doi. org/10.1016/j.indcrop.2010.12.019>. Acesso em: 16 mar. 2013. doi:10.1016/j.indcrop.2010.12.019. 